\title{
La régulation de la division cellulaire
}

La protéine kinase p34 ${ }^{\text {cde2 }}$, très conservée des levures à l'homme, joue un rôle essentiel dans le contrôle du cycle cellulaire. Elle semble agir sous la forme d'un complexe avec des cyclines, protéines dont la concentration varie au cours du cycle, à au moins deux niveaux : le déclenchement de la mitose (activité MPF, maturation... ou M-phase promoting factor) et, pendant la phase Gl, le point start qui représente vraiment l'entrée dans le cycle. Pour être active p34 cdc2 doit être déphosphorylée; elle a pour substrats diverses protéines, dont des produits d'oncogènes et, peut-être, d'anti-oncogènes ; elle est substrat elle-même de sérine/thréonine et de tyrosine kinases qui peuvent être codées par des oncogènes. D'autres gènes encore contrôlent l'activité du complexe $\mathrm{p} 34^{\mathrm{cdc} 2} / \mathrm{cycline}(\mathrm{s})$. Les récents et rapides progrès dans l'élucidation de ce système ont permis, pour la première fois, d'entrevoir les relations fonctionnelles existant entre la perception des signaux mitogènes, la mise en ouvre des produits d'oncogènes/anti-oncogènes et la mitose elle-même

\section{Christian Le Peuch}

\section{ADRESSE}

C. Le Peuch : chargé de recherche à l'Inserm. Inserm U.249 et CRBM du Cnrs, BP 5051,

a cellule, unité biologique vivante, est un système extrêmement organisé mais isolé dans lequel le désordre ne peut, thermodynamiquement parlant, qu'augmenter. Dégradation naturelle et accidents font que chaque cellule est individuellement appelée à disparaître. Pour qu'un organisme pluricellulaire puisse se maintenir en vie, il doit engendrer de nouvelles cellules au moins aussi vite que les anciennes meurent et disparaissent. La division cellulaire qui permet ce renouvellement est donc indispensable à la survie de tous les organismes. Dans un être humain adulte, par exemple, des millions de cellules doivent se diviser à chaque instant pour simplement maintenir un statu quo.

La division cellulaire et son contrôle ont toujours fasciné biologistes et biochimistes. Le phénomène de la division cellulaire lui-même peut parfaitement être observé au microscope. Il se décompose en deux étapes successives, la division nucléaire ou mitose et la division du cytoplasme ou cytodiérèse. Pour que les cellules filles soient les copies conformes de la cellule mère, il faut que cette dernière, avant sa division, ait doublé de masse et dupliqué entièrement son contenu et en particulier son matériel génétique. Ainsi les deux filles disposeront de tous les constituants dont elles ont besoin pour remplir leur(s) fonction(s) physiologique(s) éventuelle(s) ainsi que leur propre cycle de croissance et de division. Le travail de préparation à la division a lieu pendant l'interphase. Une cellule est la plupart du temps en interphase et seulement pendant une courte période en division. Pendant l'interphase, la plupart des composants cellulaires sont synthétisés de façon continue. Il est donc difficile de mettre en évidence des étapes critiques et/ou caractéristiques de la progression de la croissance cellulaire. Il faut cependant noter une exception : la synthèse de l'ADN (réplication) dans le noyau a lieu pendant une période déterminée de l'interphase que l'on a appelée phase $S$ (S pour synthèse). L'autre phase caractéristique du cycle cellulaire est la phase de division ou 
phase $\mathbf{M}$ (M pour mitose).

Ces deux phases caractéristiques sont séparées par deux périodes mal définies tant du point de vue de la biologie cellulaire que de la biochimie : la phase $\mathrm{Gl}$, entre la phase $\mathrm{M}$ et la phase $S$, et la phase $G 2$, entre la phase $S$ et la phase M (G pour gap; intervalle). Un cycle cellulaire complet comprend donc quatre phases, Gl, S, G2 et M, les phases Gl, S et G2 constituant l'interphase.

Les cellules se multiplient à des vitesses très variables et on a pu démontrer que la durée du cycle cellulaire était essentiellement conditionnée par la durée de la phase $\mathrm{Gl}$. Chez les eucaryotes, le cycle de division cellulaire contient jusqu'à trois points de régulation : (1) un point de conversion d'un état quiescent à un état prolifératif ; (2) le point d'initiation de la biosynthèse de l'ADN dans une cellule en croissance ; (3) le point d'induction de la mitose dans les cellules contenant des génomes nou- vellement répliqués.

Il est plus que probable que ces événements clés de la régulation du cycle cellulaire sont sous le contrôle de nombreux facteurs de régulation. La compréhension de la régulation du cycle cellulaire passe donc par l'identification et la caractérisation de ces différents facteurs ainsi que par la connaissance du (ou des) mécanisme(s) par le(s)quel(s) ils déclenchent une progression dans le cours du cycle cellulaire.

Ces derniers mois ont justement vu un début de description moléculaire de la régulation de la mitose. Le point remarquable de ces progrès considérables dans la compréhension des mécanismes de contrôle de la mitose est qu'ils sont l'aboutissement d'études réalisées sur des systèmes biologiques très différents et utilisant une stratégie et des techniques très différentes: la génétique des levures d'une part et la biochimie des facteurs inducteurs de la méiose ou de la mitose d'autre part, cette biochimie faisant beaucoup appel aux oufs et aux embryons d'amphibiens ou d'invertébrés marins.

\section{Le MPF, facteur de déclenchement de la méiose et de la mitose}

Les œufs fertilisés d'un amphibien, le xénope (Xenopus laevis), et d'invertébrés marins comme les étoiles de mer représentent des systèmes idéaux d'étude du cycle cellulaire. En effet leur taille est importante et, durant l'embryogenèse précoce, on observe des cycles cellulaires à la fois synchrones et rapides au cours desquels se succèdent uniquement des phases $S$ de duplication du matériel génétique et $\mathbf{M}$ de division. Les ovocytes de xénope sont arrêtés en prophase de première division méiotique, en phase G2 du cycle cellulaire. Durant la maturation, qui est induite naturellement par la progestérone,

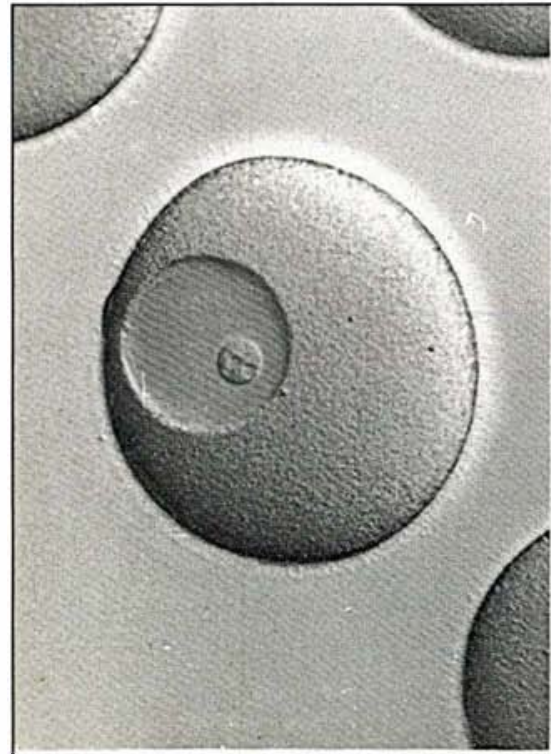

$\mathrm{t}=0$

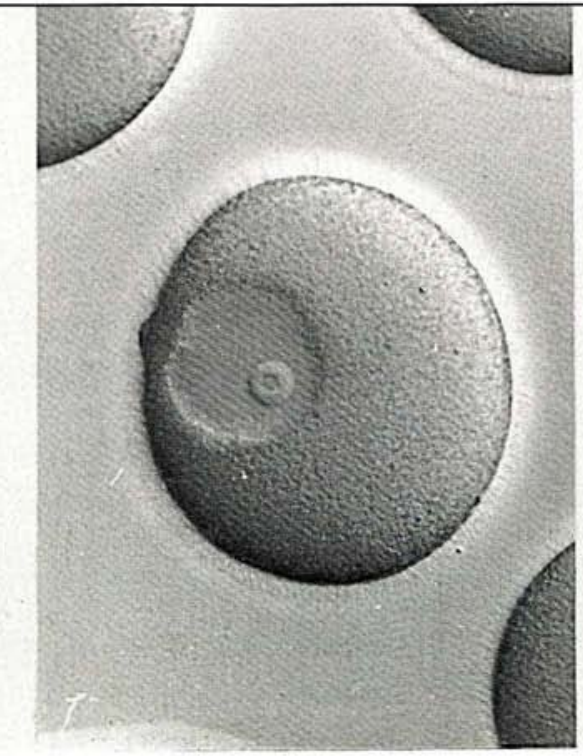

$t=15 \min$

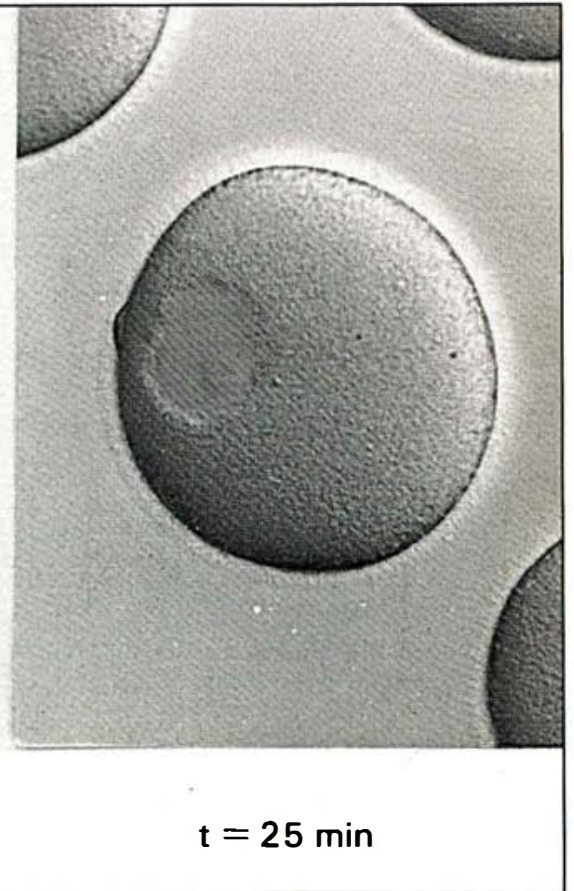

Figure 1. Rupture de la vésicule germinative d'ovocyte d'étoile de mer. Photographies prises 0,15 et 25 minutes après l'application de la 1-méthyladénine et le début de la reprise de la méiose.

$\mathrm{m} / \mathrm{s} n^{\circ} 1$ vol. 6, janvier 90 


\section{RÉFÉRENCES}

1. Masui Y, Markert CL. Cytoplasmic control of nuclear behavior during meiotic maturation of frog oocytes. J Exp Zool 1971; 177 : 129-46.

2 Kishimoto T. Regulation of metaphase by a maturation promoting factor. Dev Growth Differ $1988 ; 30: 105-15$.

3. Lohka MJ, Hayes MK, Maller JL. Purification of maturation promoting factor, an intracellular regulator of early mitotic events. Proc Natl Acad Sci USA 1988 ; 85 : 3009-13.

4. Gautier J, Norbury C, Lohka M, Nurse P, Maller JL. Purified maturation promoting factor contains the product of a Xenopus homolog of the fission yeast cell cycle gene cdc2 ${ }^{+}$. Cell 1988; 54 : 433-9.

5. Labbe JC, Lee MG, Nurse P, Picard A, Dorée M. Activation at $\mathbf{M}$ phase of a protein kinase encoded by a starfish homologue of the cell cycle control gene $c d c 2^{+}$. Nature 1988 ; $327: 251-4$.

6. Lee M, Nurse P. Cell cycle control genes in fission yeast and mammalian cells. Trends Genet $1988 ; 4$ : 287-90.

7. Hindley J, Phear GA. Sequence of the cell division gene $c d c 2$ from Schizosaccharomyces pombe; patterns of splicing and homology to protein kinases. Gene 1984; 31 : 129-34.

8. Simanis V, Nurse P. The cell cycle control gene $c d c 2^{+}$of fission yeast encodes a protein kinase potentially regulated by phosphorylation. Cell 1986; 45 : 261-8.

9. Lee MG, Nurse P. Complementation used to clone a human homolog of the fission yeast cell cycle control gene $c d c 2^{+}$. Nature 1987 ; 327 : 31-5.

10. Draetta G, Brizuela L, Potashkin J, Beach D. (1987) Identification of p34 and pl3, human homologs of the cell cycle regulators of sission yeast encoded by $c d c 2^{+}$and $s u c 1^{+}$. Cell 1987 ; $50: 319-25$.

11. Draetta G, Beach D. Activation of cdc2 protein kinase during mitosis in human cells; cell cycle dependent phosphorylation and subunit rearrangement. Cell 1988; 54 : 17-26.

12. Gautier J, Matsukawa T, Nurse P, Maller J. Dephosphorylation and activation of Xenopus p34cdc2 protein kinase during the cell cycle. Nature 1989 ; 339 : 626-9.

13. Labbé JC, Picard A, Peaucellier g, Cavadore JC, Nurse P, Dorée M. Purification of MPF from starfish : identification as the $\mathrm{Hl}$ histone kinase $\mathrm{p} 34^{\mathrm{cdc} 2}$ and a possible mechanism for its periodic activation. Cell 1989; l'ovocyte devient un œuf non fécondé en terminant sa première division méiotique et en s'arrêtant ensuite en métaphase II. Cette maturation peut être facilement détectée grâce à la rupture de l'enveloppe nucléaire ou GVBD (germinal vesicle breakdown) qui suit de peu de temps la reprise du cycle cellulaire (figure 1, p. 11)
En 1971, on a pu montrer que la maturation d'un ovocyte de xénope pouvait être induite par la microinjection de cytoplasme prélevé dans des ovocytes en phase M[1]. Le facteur responsable de la maturation fut appelé MPF (maturation promoting factor). On devait par la suite montrer que ce facteur ne présente pas de spécificité d'espèce et peut aussi bien

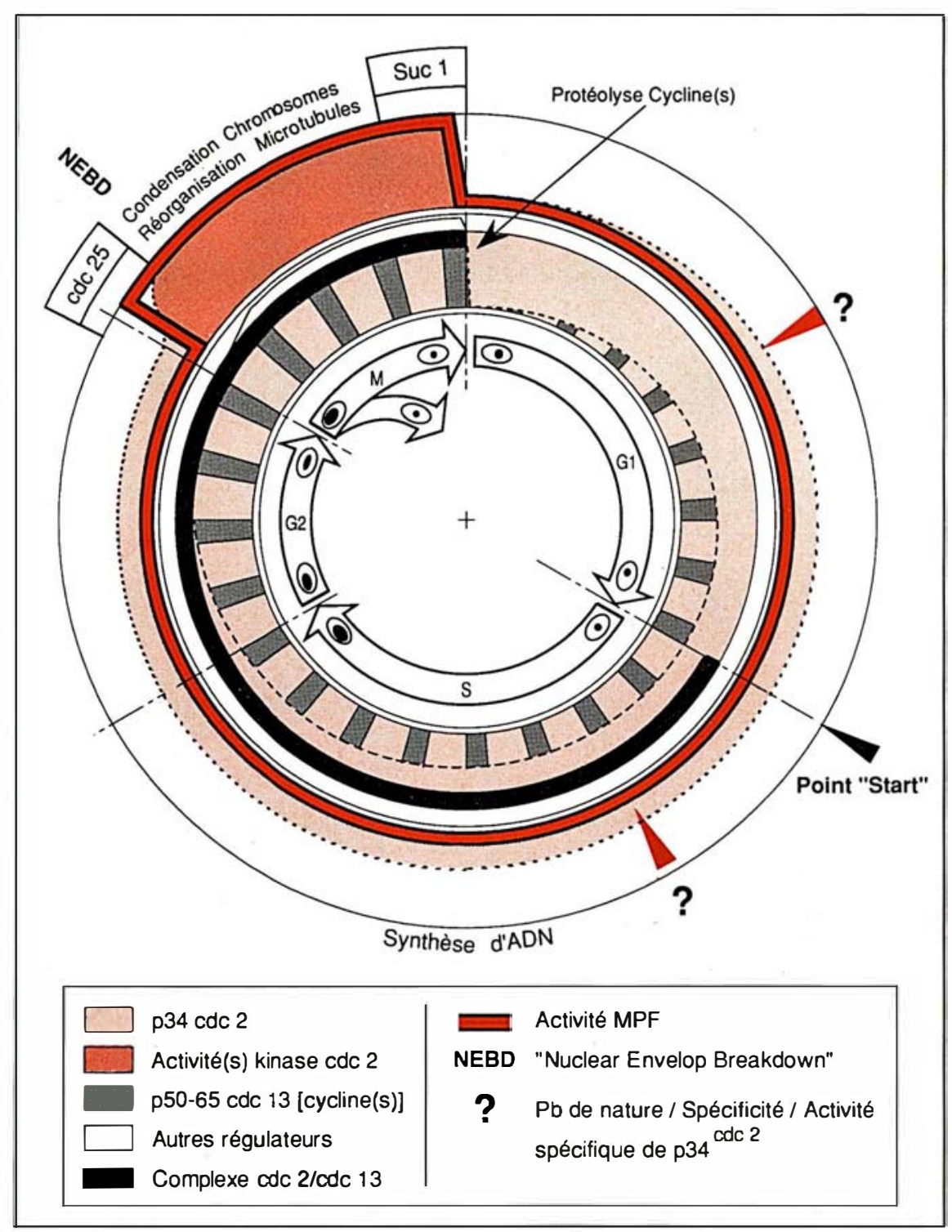

Figure 2. Représentation schématique du cycle cellulaire. La présence des différents facteurs et activités qui interviennent dans sa régulation est symbolisée par l'épaisseur de la bande correspondante. 
induire la méiose dans des ovocytes que la mitose dans des cellules somatiques [2].

L'activité MPF varie de façon cyclique au cours des cycles méiotiques ou mitotiques et montre un maximum en métaphase (figure 2). Cette activité est très difficile à purifier. Des purifications de 20 à 200 fois ont été rapportées dans la littérature, la reprise de la méiose d'ovocytes micro-injectés constituant alors le test d'activité MPF. Récemment, un test plus sensible a été mis au point qui utilise des noyaux purifiés dans un système acellulaire. L'utilisation de cette technique au long d'une purification assez lourde a permis de purifier le MPF 3000 fois [3]. Les fractions enrichies en activité MPF contenaient également des protéines (p32 et p45) de 32 et $45 \mathrm{kDa}$ de poids moléculaire. D'autre part, on trouvait également dans ces fractions une activité protéine sérine-thréonine kinase, capable de phosphoryler la p45 endogène ainsi que l'histone $\mathrm{Hl}$, la caséine et l'inhibiteur 1 des phosphatases. Il a été ensuite démontré que la protéine p32 était la protéine kinase [4].

D'autres recherches, menées dans le but de purifier la protéine kinase contenue dans le MPF d'étoile de mer, aboutissaient à la purification d'une protéine de $34 \mathrm{kDa}$ de poids moléculaire [5].

En 1987, il a été montré qu'il existe dans le génome humain des gènes capables de remplacer les gènes $c d c 2$ mutants de levures. La protéine humaine codée par ce gène doit donc être pratiquement identique à celle de levure et certains des mécanismes de la division cellulaire conservés de la levure à l'homme. Cette protéine p34 ${ }^{\text {cdc2 }}$ de $34 \mathrm{kDa}$ de poids moléculaire est, comme nous le verrons plus loin, une protéine kinase, homologue des p34 (ou p32) de xénope ou d'étoile de mer.

\section{La génétique des levures}

Chez les champignons que sont les levures, il est possible d'utiliser des stratégies génétiques extrêmement puissantes pour élucider des problèmes de biologie moléculaire ou cellulaire. Les résultats obtenus ne sont malheureusement pas toujours $\mathrm{m} / \mathrm{s} n^{\circ} 1$ vol. 6 , janvier 90 applicables aux cellules de mammifères. Les techniques de manipulation des gènes de mammifères par mutagenèse sont également très périlleuses. En fait, les chercheurs ont utilisé la possibilité, évoquée plus haut, de transférer des gènes entre des cellules de levures et des cellules de mammifères, et donc d'appliquer les techniques génétiques utilisées dans l'étude des levures à des cellules de mammifères [6]. Chez la levure Schizosaccharomyces pombe, la division intervient dès que la cellule atteint une certaine longueur. Chez certains mutants, à une température donnée, la division ne se fera plus, alors que la croissance continuera. Ces mutations sont dues à un groupe de gènes, les gènes $c d c$ (cell division cycle); il existe d'autres mutants, dits de type wee qui se divisent avant d'avoir atteint la taille «requise ». Deux points de contrôle majeurs interviennent dans le déroulement du cycle de division de la levure $S$. pombe. Le premier est situé en fin de phase $\mathrm{Gl}$, au point connu comme point de restriction ou $\mathrm{R}$ ou encore start. Lorsque la cellule a dépassé ce point, elle accomplit le reste du cycle mitotique et la voie alternative de conjugaison ne peut intervenir qu'une fois que le cycle engagé aura été achevé. Les fonctions cellulaires codées par les deux gènes $c d c 2^{+}$et $c d c 10^{+}$sont nécessaires pour dépasser le point $\mathrm{R}$. Le second point se situe en fin de phase G2 et contrôle l'entrée en mitose en fin de cycle cellulaire. Les produits codés par les quatre gènes $c d c 2^{+}, w e e 1^{+}, c d c 25^{+} \mathrm{et}$ nim $1^{+}$semblent responsables de cette régulation.

Il semble donc que le gène $c d c 2^{+}$, ou tout au moins le produit pour lequel il code, soit très important dans la régulation du cycle cellulaire car il intervient dans les régulations du passage au travers du point start (ou R) et de l'entrée en mitose.

\section{p34cdc2}

Le gène $c d c 2^{+}$qui contient quatre introns, code pour une protéine kinase de $34 \mathrm{kDa}$ de poids moléculaire qui présente de nombreuses homologies avec d'autres protéines kinases [7]. En fait, des anticorps dirigés contre le produit du gène $c d c 2^{+}$reconnaissent une phospho- protéine de 34 kDa (p34cdc2) qui possède une activité protéine kinase in vitro [8]. Toutes les espèces eucaryotes qui ont été testées possèdent une protéine kinase homologue au produit du gène $c d c 2^{+}$de $S$. pombe, qu'il s'agisse de $S$. cerevisiae, où le gène $C D C 28$ code pour une protéine très similaire, ou d'eucaryotes multicellulaires tels que l'étoile de mer, la palourde, le xénope, le rat, la souris ou l'homme. Il faut noter que toute mutation qui touche l'activité kinase de $\mathrm{p} 34$, détruit l'activité du gène $c d c 2^{+}$.

Les protéines codées par les gènes $c d c 2$ de différentes espèces et $C D C 28$ de $S$. cerevisiae présentent environ $60 \%$ d'homologie les unes par rapport aux autres. On trouve dans toutes ces séquences une suite de 16 acides aminés qui est intégralement conservée, la séquence PSTAIR (EGVPSTAIREISLLKE) [9]. De façon assez surprenante, cette séquence ne se situe pas dans le domaine consensus des protéines kinases et pourrait plutôt être impliquée dans la liaison de ces kinases à des protéines régulatrices. On sait depuis longtemps que le déclenchement de la méiose ou de la mitose est marqué par la stimulation de la phosphorylation de nombreuses protéines intracellulaires. Quoique la plupart des phosphoprotéines ne soient pas identifiées, on sait que les lamines (protéines constitutives de la membrane nucléaire), la vimentine (protéine du cytosquelette), les histones $\mathrm{H} 3$ et $\mathrm{S} 6$, protéine de la petite sous-unité ribosomale, sont phosphorylées. Enfin, la phosphorylation des histones $\mathrm{Hl}$ est caractéristique de l'état de prolifération des cellules.

L'ensemble des résultats rapportés cidessus tend à confirmer que la phosphorylation de certaines protéines jouerait un rôle déterminant dans le contrôle et la régulation du cycle cellulaire.

\section{Activités protéine kinase et division cellulaire}

Les histones $\mathrm{Hl}$ ou la caséine ont été souvent utilisées comme substrats pour tester in vitro l'activité kinase de p34cdc2 ; cependant, in vivo, les substrats ainsi que le rôle des phosphorylations catalysées par cette 


\section{RÉFÉRENCES}

14. Russell P, Nurse P. cdc $25^{+}$functions as an inducer in the mitotic control of fission yeast. Cell 1986; 45 : 145-53.

15. Russell $P$, Nurse $P$. Negative regulation of mitosis by wee1 ${ }^{+}$, a gene encoding a protein kinase homolog. Cell 1987 ; 49 : 559-67.

16. Russell $P$, Nurse $P$. The mitotic inducer niml ${ }^{+}$function in a regulatory network of protein kinase homologs controlling the initiation of mitosis. Cell $1987 ; 49: 569-76$

17. Moreno S, Hayles J, Nurse P. Regulation of p34cdc2 protein kinase during mitosis. Cell 58, 361-372. 1989 ; 58 : 361-72.

18. Booher RN, Alfa CE, Hyams JS, Beach $\mathrm{DH}$. The fission yeast cdc2/cdcl3/sucl protein kinase: Regulation of catalytic activity and nuclear localization. Cell 1989 ; 58: 48597.

19. Brizuela L, Draetta G, Beach D. pl3 sucl acts in the fission yeast cell division cycle as a component of the p34cdc2 protein kinase. EMBO J 1987 ; 6 : 3507-14.

20. Beach D, Durkacz B, Nurse P. Functionaly homologous cell cycle control genes in budding and fission yeast. Nature $1982 ; 300$ : 706-9.

21. Solomon $\mathbf{M}$, Booher $r$, Kirschner $\mathbf{M}$, Beach D. Cyclin in fission yeast. Cell 1988; $54: 738-9$.

22. Goebl M, Byers B. Cyclin in fission yeast. Cell 1988; 54 : 739-40.

23. Picard A, Peaucellier G, Le Bouffant F, Le Peuch CJ, Dorée M. Role of protein synthesis and proteases in production and inactivation of maturation promoting activity during meiotic maturation of starfish oocytes. Dev Biol 1985 ; 109 : 311-20.

24. Murray AW, Kirschner MW. Cyclin synthesis drives the early embryonic cell cycle. Nature 1989 ; 339 : 275-80.

25. Murray AW, Solomon MJ, Kirschne MW. The role of cyclin synthesis and degradation in the control of maturation promoting factor activity. Nature $1989 ; 339: 280-6$.

26. Labbé JC, Capony JP, Caput D, et al. MPF from starfish oocytes at first meiotic metaphase is an heterodimer containing one molecule of cdc2 and one molecule of cyclin B. EMBO J 1989; 8: 3053-8.

27. Meijer L, Arion D, Golsteyn R, et al. Cyclin is a component of the sea urchin egg M-phase specific histone $\mathrm{Hl}$ kinase. EMBO J $1989 ; 8: 2275-82$

28. Hayles J, Nurse P. Cell cycle regulation kinase sont encore mal connus.

Nous avons vu que $\mathrm{p} 34^{\mathrm{dc} 2}$ est une phosphoprotéine; l'activité kinase de $\mathrm{p} 34^{\text {ddc2 }}$ de même que son état de phosphorylation peuvent être corrélés à l'état prolifératif des cellules: quand les cellules de $S$. pombe sont métaboliquement bloquées avant le point start, p34cdc2 ne possède plus d'activité protéine kinase et n'est plus phosphorylée [8]. Le retour à un état prolifératif sera accompagné d'une phosphorylation de $\mathrm{p} 34^{\text {dcc } 2}$ et d'une restauration de son activité kinase. Ceci a été confirmé dans d'autres systèmes où le niveau de concentration de p34 cdc2 ne change pas non plus pendant le cycle cellulaire alors que son activité kinase varie [10-13] (figure 2, p. 12). Ainsi, chez $S$. cerevisiae, l'activité kinase de p34 $4^{\mathrm{cdc} 28}$ atteint son plus haut niveau en $\mathrm{Gl}$ alors que l'activité kinase de p34 cdc2(Hs) atteint son plus haut niveau en fin de phase G2 et en mitose dans les cellules HeLa. Ces augmentations d'activité protéine kinase s'accompagnent de la formation de complexes de haut poids moléculaire entre p34 et d'autres protéines.

Ainsi l'activité protéine kinase de p34 cdc2 pourrait contrôler l'entrée en phase $S$ et l'entrée en mitose au cours du cycle cellulaire par phosphorylation de protéines cibles dont la définition est fondamentale si nous voulons comprendre complètement les mécanismes de contrôle du cycle cellulaire. Il convient également de comprendre comment, et par quoi, est contrôlée l'activité kinase de p34 $4^{\mathrm{cd} c 2}$.

Nous avons vu précédemment qu'au moins trois autres gènes sont impliqués dans le contrôle de l'initiation de la mitose, il s'agit de wee ${ }^{+}$, cdc25+ et niml $^{+}[14-16] ;$ weel $1^{+}$et nim $1^{+}$codent pour des protéines ayant une activité protéine kinase et des homologies avec les séquences consensus des protéine kinases connues.

La surexpression du produit de $c d c 25^{+}$induit la mitose et, réciproquement, la délétion du gène induit un blocage de la mitose. Il semble en fait que la transition G2/M et l'activation du complexe $\mathrm{p} 34^{\mathrm{cdc} 2}-\mathrm{p} 63^{\mathrm{cdc} 19}$ requiert la présence du produit du gène $c d c 25[17,18]$. Dans le cas de wee $1^{+}$, c'est exactement l'opposé qui est observé. Le gène wee $1^{+}$coderait donc pour une protéine inhibitrice de l'induction de la mitose. Enfin, l'expression du troisième gène nim $1^{+}$ conduit à une reprise de la mitose, ce qui suggère qu'il code pour un inducteur de la mitose. En fait, l'effet inducteur de niml $^{+}$ne peut être observé que si le gène wee ${ }^{+}$est exprimé, ce qui semble indiquer que le produit de $n i m 1^{+}$agit en amont du produit de $w e e 1^{+}$en bloquant l'effet inhibiteur de ce dernier sur p34 $4^{\text {cdc2 }}$ (figure 3, p. 16).

Les mutations de $c d c 2^{+}$qui conduisent à la formation de $\mathrm{p} 34^{\mathrm{cd} c 2}$ capable de provoquer une induction de la mitose de manière constitutive appartiennent à deux classes : celles qui conduisent à une p34 dont l'activité ne dépend plus de l'expression du gène wee ${ }^{+}$et celles qui conduisent à la formation d'une p34 dont l'activité est indépendante du niveau d'expression du gène $c d c 25^{+}$. Les produits de ces deux gènes agissent donc probablement en amont de p34. Sachant que, comme nous l'avons vu plus haut, wee $1^{+}$et niml $^{+}$codent pour des protéine kinases, la régulation de la mitose pourrait mettre en jeu des séquences d'activités kinases niml/weel/cdc2 (figure 3). Les gènes niml $^{+}$et wee $^{+}$codent respectivement pour des protéines de $42 \mathrm{kDa}$ et $96 \mathrm{kDa}$. Quant au gène $c d c 25^{+}$, il code pour une protéine de $65 \mathrm{kDa}$.

D'autres gènes semblent interagir avec les voies de régulation décrites ci-dessus : ainsi, l'expression de $\mathrm{sucl}^{+}$ permet à des mutants thermosensibles de $c d c 2^{+}$de pousser à des températures non permissives. Des observations, d'abord génétiques, ont conduit à penser qu'il devait y avoir une interaction physique directe entre $\mathrm{p} 34^{\mathrm{dcc} 2}$ et le produit $\operatorname{suc} \mathrm{I}^{+}[10$, 19]. Ceci a été confirmé par des expériences utilisant des anticorps dirigés contre la protéine de $13 \mathrm{kDa}$, supposée, à partir de l'analyse de la séquence du gène, comme étant codée par le gène $\mathrm{sucl}^{+}$. Ces anticorps lient bien une protéine de $13 \mathrm{kDa}$, confirmant ainsi l'hypothèse faite un peu plus haut, mais aussi un pourcentage significatif de $\mathrm{p} 34^{\mathrm{dc} c}$. Il y aurait donc bien une interaction physique entre $\mathrm{p} 34^{\text {cdc2 }}$ et $\mathrm{pl} 3^{\text {sucl }}[10]$. Un peptide homologue de $\mathrm{pl} 3^{\text {sucl }}$ a été identifié chez l'homme et on a pu montrer qu'il formait des complexes 
avec la protéine $\mathrm{p} 34^{\mathrm{cdc} 2(\mathrm{Hs})}$. Le peptide pl3 $3^{\text {sucl }}$ est également un inhibiteur de l'activité MPF. Cette inhibition présente la propriété d'être levée en présence de concentrations élevées de peptide PSTAIR, ce qui suggère que cette séquence jouerait un rôle clé dans les interactions de p34 avec des protéines régulatrices. Enfin, et nous reviendrons sur ce point un peu plus loin, pl3 serait impliquée dans la régulation de la phosphorylation de p34 sur des résidus tyrosine, cette phosphorylation étant elle-même impliquée dans la régulation de l'activité kinase de p34.

\section{Les cyclines}

Le produit du gène $c d c 13$ serait quant à lui capable de suppléer à des mutations de $c d c 2^{+}$qui toucheraient uniquement le second point de contrôle de l'entrée en mitose [20]. Il a été montré que la séquence du produit de $c d c 13$ de levure présentait un haut degré d'homologie avec celles de différentes cyclines [21, 22] qui ont été décrites dans plusieurs espèces animales, et en particulier chez le xénope et l'homme comme des protéines de respectivement 45 et $62 \mathrm{kDa}[4,11]$. Dans les cellules en division, la synthèse de protéines au cours d'un cycle est nécessaire au déclenchement du cycle mitotique suivant. Cette synthèse concerne probablement un ou des éléments régulateurs importants du cycle cellulaire, car les embryons aux stades précoces contiennent des quantités importantes des enzymes et protéines requises pour la synthèse d'ADN et la mitose. Les cyclines pourraient être le (ou un des) régulateur de l'entrée en mitose.

Les cyclines sont en effet des protéines qui s'accumulent durant l'interphase et dont la quantité décroît brusquement, du fait de leur protéolyse, lors de la mitose (figure 2). En fait, le temps de demi-vie des cyclines est long durant l'interphase et considérablement raccourci durant la mitose, la synthèse ne semblant soumise à aucune régulation. Les premières indications que les cyclines jouaient un rôle dans la régulation de la mitose sont venues de l'observation que l'injection d'ARNm de cycline déclenchait la maturation d'ovocytes de xénopes.

$\mathrm{m} / \mathrm{s} n^{\circ} 1$ vol. 6 , janvier 90
De même, il semble que la dégradation des cyclines soit nécessaire à l'inactivation du MPF et à la sortie de la méiose ou de la mitose. En effet, les agents pharmacologiques qui augmentent la durée de la mitose stabilisent les cyclines; de plus, en présence de hautes concentrations d'inhibiteurs de protéases microinjectés, les ovocytes d'étoile de mer s'arrêtent en méiose I, et ceci pourrait être la conséquence de l'inhibition de la dégradation des cyclines [23]. Des études très récentes [24-25] ont montré qu'une cycline mutée résistante à la protéolyse empêchait l'inactivation du MPF et la sortie de mitose in vivo et in vitro. Les mêmes auteurs ont montré que la cycline était dans leurs conditions expérimentales un facteur nécessaire et suffisant pour contrôler l'activité du MPF.

En fait, il a été récemment montré [26] que le MPF d'étoile de mer en métaphase de première division de méiose est un hétérodimère formé d'une molécule de p34 ${ }^{\text {cdc2 }}$ et d'une molécule de cycline B (figure 2). Des résultats similaires ont été obtenus chez l'oursin [27]. La cycline est-elle pour autant seule responsable de la régulation de l'activation de p34cdc2 - et par là du MPF - et si oui par quel mécanisme? Sachant que pour être active, p34 doit être déphosphorylée (figure 3), un certain nombre de mécanismes potentiels peuvent être avancés : (a) une simple liaison de la cycline à p34 conduirait à la formation de l'hétérodimère actif ; (b) la liaison de la cycline à p34 permettrait la déphosphorylation de cette dernière par une phosphatase et son activation ; rien n'empêche de penser que la cycline pourrait être la phosphatase de p34cdc2 ; (c) l'association de la cycline à p34 empêcherait sa phosphorylation et permettrait ainsi l'accumulation de p34 actif dans le complexe avec la glycine, sans variation d'activité phosphatase.

\section{Les substrats de p34cdc2}

Ainsi que nous l'avons souligné plus haut, le rôle des phosphorylations catalysées par p34cdc2 est assez mal connu. L'association de la kinase avec les fractions nucléaires ou avec les centrosomes suggère que des protéines appartenant à ces systèmes pourraient être phosphorylées, on peut penser en particulier aux lamines et aux histones - impliquées dans l'organisation du nucléosquelette et de la chromatine - qui subissent des cycles de phosphorylation-déphosphorylation pendant le cycle cellulaire [28]. On sait que les histones, $\mathrm{Hl}$ notamment, sont de bons substrats de p34. De même la protéine ribosomale S6 est phosphorylée par p34. On sait également que la cycline, $\mathrm{p} 56^{\mathrm{cdc} 13}$ ou $\mathrm{p} 63^{\mathrm{cdc} 13}$, est un substrat de p34 sans savoir quel est le rôle de cette phosphorylation dans la régulation de l'activité MPF. Il a été récemment montré que dans l'ovocyte de xénope, une protéine de $47 \mathrm{kDa}$ de poids moléculaire est phosphorylée in vitro et in vivo par p34 [29]. Cette phosphoprotéine p47, qui est purifiée sous forme d'un hétéropolymère de haut poids moléculaire comprenant deux autres protéines p36 et p30, est présente dans les ovocytes en prophase et dans les ovocytes matures. L'analyse des séquences d'acides aminés de p47 et de p30 montre que ces protéines ont des analogies structurales avec la sous-unité $\beta$ de facteurs d'élongation des chaînes polypeptidiques comme EFl. L'ARN polymérase II eucaryotique active possède un domaine carboxy-terminal répétitif très phosphorylé; il semble que p34cdc2 soit responsable de la phosphorylation de résidus sérine dans ce domaine, ce qui amène à supposer que cette kinase jouerait un rôle dans la régulation de la transcription [30]. Ainsi quelques substrats commencent à être décrits, mais ces phosphorylations doivent être reliées à des régulations de fonctions cellulaires et beaucoup reste donc à faire dans ce domaine, d'autant que des produits d'oncogènes cellulaires apparaissent également être des substrats de p34.

\section{p34cdc2 et tyrosine kinases}

Deux publications récentes montrent que l'un des substrats de la protéine kinase associée à p34cdc2 pourrait être p60 ${ }^{\text {c-src }}$, le produit du proto-oncogène src [31, 32]. En effet, le MPF extrait d'ovocytes de xénopes phosphoryle p60 ${ }^{\text {c-src }}$ sur les Thr 34 et 46 ainsi que sur la Ser 72. Or ces sites sont ceux qui sont phosphorylés dans les cellules NIH 3T3 en mitose. Pratiquement les mêmes résultats ont été 


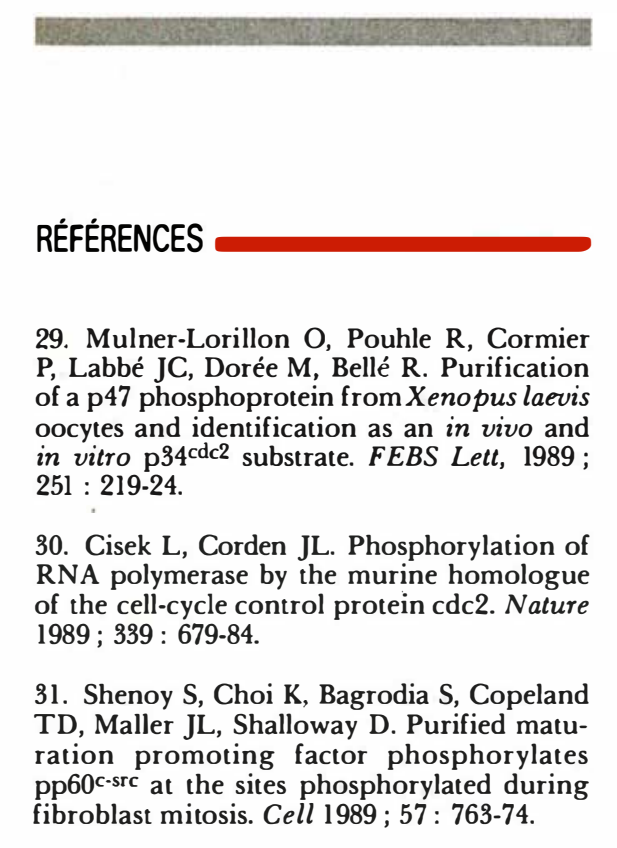

32. Morgan DO, Kaplan JM, Bishop JM, Varmus HE. Mitosis-specific phosphorylation of p60 $0^{\text {c-src }}$ by p34cdc2-associated protein kinase. Cell $1989 ; 57$ : 775-86.

33. Morla AO, Draetta G, Beach D, Wang JYJ. Reversible tyrosine phosphorylation of cdc2 : dephosphorylation accompanies activation during entry into mitosis. Cell 1989 ; 58 : 193-203.

34. Dunphy WG, Newport JW. Fission yeast pl3 blocks mitotic activation and tyrosine dephosphorylation of the Xenopus $c d c 2$ protein kinase. Cell 1989; 58 : 181-9l.

35. De Caprio JA, Ludlow JW, Lynch D, et al. The product of the retinoblastoma susceptibility gene has properties of a cell cycle regulatory element. Cell $1989 ; 58$ : 1085-95.

36. Buchkovich K, Duffy LA, Harlow E. The retinoblastoma protein is phosphorylated during specific phases of the cell cycle. Cell $1989 ; 58$ : 1097-1105.

37. Chen PL, Scully P, Shew JY, Wang JYJ, Lee WH. Phosphorylation of the retinoblastoma gene product is modulated during the cell cycle and cellular differentiation. Cell 1989 ; 58 : 1193-8.

38. McVey D, Brizuela L, Mohr I, Marshak DR, Gluzman Y, Beach D. Phosphorylation of large tumour antigen by $c d c 2$ stimulates SV40 DNA replication. Nature 1989 ; 341 : 503-7.

39. Sagata N, Oskarsson M, Copeland T, Brumbaugh J, Van de Woude GF. Function of $c$-mos proto-oncogene product in meiotic maturation in Xenopus oocytes. Nature 1988 ; 335 : 519-25.

40. Sagata N, Daar L, Oskarsson M, Showalter SD, Van de Woude GF. The product of the mos proto-oncogene as a candidate « initiator » for oocyte maturation. Science 1989 ;

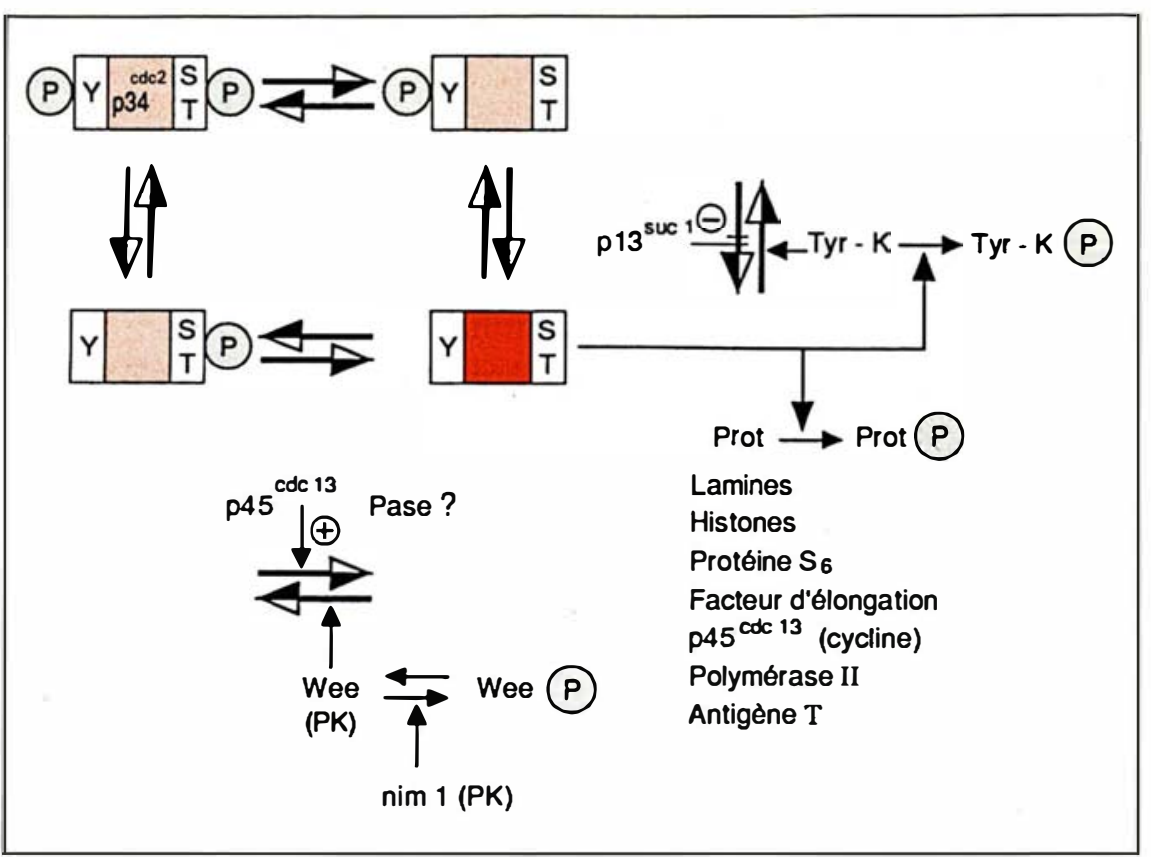

Figure 3. Représentation des interconversions entre les différents états de phosphorylation de cdc2. Les directions des différentes réactions sont reproduites en bas (phosphorylation sur des sérines et des thréonines) et sur la droite (phosphorylation sur des tyrosines) avec les facteurs qui les catalysent ou qui les régulent. $Y=$ tyrosine $; S=$ sérine $;=$ thréonine $P=$ acide phosphorique.

obtenus dans des cellules HeLa. Cependant, si l'on sait que l'activité tyrosine kinase de p60 $0^{\text {-src }}$ est augmentée dans les cellules en mitose, aucune démonstration n'a été faite que les phosphorylations de $\mathrm{p} 60^{\text {cssc }}$ par $\mathrm{p} 34^{\mathrm{cdc} 2}$ décrites plus haut augmentaient son activité tyrosine kinase. Il reste donc à découvrir quel est le mécanisme par lequel p34cdc2 pourrait intervenir au niveau de proto-oncogènes qui seraient impliqués dans la division et la différenciation cellulaires.

Les interactions entre p34 et tyrosine kinase(s) ont été rendues encore plus complexes par la découverte que l'activité de p34 était contrôlée par sa phosphorylation sur des tyrosines [33-34]. En fait, le niveau de phosphorylation de p34 sur des tyrosines est élevé en interphase et nul pendant la mitose, aussi bien dans l'ovocyte de xénope que dans des fibroblastes de souris 3T3. Dans les fibroblastes 3T3, cette phosphorylation s'accompagne de la formation d'un complexe p34/p62. En fait, une déphosphorylation de p34 par une phosphotyrosine phosphatase serait une des étapes nécessaire à l'activation de l'activité kinase de p34. Cette déphosphorylation aurait lieu juste avant l'entrée en mitose et serait contrôlée par pl3suc (figure 3). En effet, le produit du gène suc est connu pour inhiber l'entrée en mitose; pl3 pourrait ainsi bloquer la déphosphorylation des phosphotyrosines de p34 et donc son activation [34].

Il apparaît donc que le déclenchement et le contrôle de l'entrée en mitose - ou en méiose - de cellules en interphase impliquerait de nombreuses protéine kinases, aussi bien des tyrosine kinases que des sérinethréonine kinases. Parmi ces dernières, $\mathrm{p} 34^{\mathrm{dc} 2}$ jouerait un rôle clé. Il semble en effet non seulement qu'elle soit la cible de nombreuses voies de régulation, mais aussi qu'elle ait de nombreuses cibles dont elle pourrait contrôler l'activation ou la fonction. 
Cependant, il convient ici de souligner plusieurs points importants : (a) si p34 cdc2 est un dénominateur commun à la reprise de la méiose ou de la mitose depuis la levure jusqu'à l'homme, des différences interespèces apparaissent pour ce qui concerne les autres protagonistes du système régulateur de la division cellulaire; (b) les activités kinase et phosphatase semblent jouer une fois de plus un rôle déterminant, il semble aussi que la localisation de certains éléments du système soit un facteur de régulation (18); (c) les effets de p34cdc2 sont pléiotropes, ce qui implique que de nombreux substrats voient leur état de phosphorylation modifié lors de l'activation de la kinase. Ainsi, il a été montré que le produit d'un antioncogène, la protéine pl05 ${ }^{\mathrm{Rb}}$ du rétinoblastome est phosphorylée de façon cyclique au cours du cycle cellulaire [35-37]; il y a une probabilité non négligeable, fondée en particulier sur des analyses de séquence, pour que cette protéine soit un substrat de p34 ${ }^{\text {cdc2 }}$, comme l'est l'antigène tumoral $T$, dont la phosphorylation par p34 conduit à une stimulation de la réplication de l'ADN de SV40 [38]; (d) enfin, il ne faut pas perdre de vue que si p34 ${ }^{\text {cdc2 }}$ représente un carrefour important dans la régulation du cycle cellulaire, l'activation de p34 n'est que l'aboutissement de cascades de réactions convergentes et que les premières des étapes qui vont conduire de la réception d'un signal au déclenchement de la division cellulaire sont encore bien mal connues. Dans ce contexte, il est tout à fait intéressant de noter que le produit du protooncogène $c$-mos, $p p^{39 m o s}$, serait impliqué de façon plus précoce que p34 dans la maturation des ovocytes de xénopes [39, 40].

Ainsi, peu à peu, nous comprenons mieux comment se fait le déclenchement du processus de division cellulaire; cependant beaucoup reste à faire pour appréhender tous les mécanismes mis initialement en jeu ainsi que les régulations fines et spécifiques des processus de méiose et de mitose dans toutes les espèces. Restera, lorsque ces études auront progressé, à comprendre les perturbations pouvant conduire aux anomalies de la division cellulaire, c'est-à-dire avant tout au cancer

$\mathrm{m} / \mathrm{s} n^{\circ} 1$ vol. 6 , janvier 90

\section{Summary}

Regulation of cell division

In the course of the past few years, genetic studies on yeasts and biochemical studies on systems such as oocytes from amphibians and echinoderms have finally come together to give us the beginning of an explanation for the mechanism of regulation of cell division. In this mechanism, the product of the gene $c d c 2, p 34$ cdc2, which is a protein kinase, plays a key role. The activity of this protein kinase is itself regulated by protein tyrosine kinase(s) and protein serine-threonine kinases such as the products of the genes wee $1^{+}$and nim $^{+}$. p34 cdc2 interacts as well with other proteins (pl3sucl, p45 cdc13) which regulate its state of phosphorylation and, as a consequence, its activity. The substrates of p34 are also starting to become known. Among these are histone $\mathrm{Hl}$, the ribosomal protein S6, the elongation factor EFl, polymerase II and the product of the cellular oncogene src, p60c-src. Although our understanding of this area is progressing very quickly, much is still left to do to enable us to have a complete understanding of the mechanism of regulation of cell division.

\section{TIRÉS A PART}

C. Le Peuch. 